Article

\title{
Facile Fabrication of Micro/Nano Hierarchical SERS Sensor via Anisotropic Etching and Electrochemical Treatment for Malachite Green Detection
}

\author{
Chu-Yu Huang *D and Chih-Hung Chien \\ Department of Mechanical Engineering, National Chung Hsing University, Taichung 402, Taiwan; \\ as6659871@gmail.com \\ * Correspondence: tomhuang@nchu.edu.tw
}

Received: 18 November 2019; Accepted: 29 November 2019; Published: 2 December 2019

Featured Application: The SERS active substrates developed in this study have great potential in areas such as medicine, food safety, and biotechnology.

\begin{abstract}
We propose a facile method to produce micro/nano hierarchical surface-enhanced Raman scattering (SERS) active substrates using simple steps and inexpensive costs. The proposed SERS substrate is a silicon pyramid array covered by a nanostructured gold film (AuNS @ SiPA). Through finite element method (FEM) simulation, we showed that many strong local electric field enhancements (hot spots) were formed between the nano-gap of gold nanostructures. In addition, the micron-scale pyramid structure not only increases the sensing surface area of the sensor, but also helps trap light. By combining these micro and nano structures, the proposed micro/nano hierarchical SERS sensor exhibited high sensitivity. Experimental results confirmed that the AuNS @ SiPA substrate has high sensitivity. The SERS signal enhancement factor obtained from the Rhodamine 6G (R6G) probe molecules was as high as $1 \times 10^{7}$ and the SERS substrates were found to be able to detect a very low concentration of $0.01 \mathrm{nM}$ malachite green (MG) solution. Therefore, this study provides a novel and practical method for fabricating SERS substrates that can facilitate the use of SERS in medicine, food safety, and biotechnology.
\end{abstract}

Keywords: SERS; surface-enhanced Raman scattering; gold nanostructure; micro/nano hierarchical; micro-pyramid array; malachite green; MG

\section{Introduction}

Malachite green is often illegally used in aquaculture to prevent aquatic animals from being infected by parasites, fungi, and protozoa [1]. According to the results of animal experiments, malachite green can poison the liver of experimental animals, cause anemia and hyperthyroidism, and cause lung adenomas of experimental rats [2]. In order to avoid the harm of malachite green to human health, malachite green is prohibited from being used for aquaculture animals in the United States, and the UK [3]. According to the "Standards for Residues of Animal Drugs" of the Department of Health and Welfare, the residue of malachite green in aquatic products is "not allowed to be detected". The European Commission requires that detection methods be able to determine the sum of MG at the minimum performance limit of $2 \mathrm{ng} / \mathrm{g}$ (approximately $5.5 \mathrm{nM}$ ) [4]. The detection of malachite green residues is usually carried out at a sophisticated Laboratory using expensive equipments, such as liquid chromatography visible detection (LC-VIS) for routine screening and liquid chromatography mass spectrometry (LC-MS) for residue confirmation [1]. It is a time consuming and expensive process. There is a need for high throughput and cost-effective methods for screening these aquaculture products. 
Surface-enhanced Raman spectroscopy (SERS) technology has the potential to provide a very fast and sensitive method of detecting chemicals and biomolecules. It can be a solution for this unmet need.

The 3D microstructure of the SERS substrate has a significant impact on the SERS signal. Studies have shown that the SERS signal can be enhanced by changing the 2D planar substrate into a 3D microstructure substrate, for example, silicon nanowire [5], and pyramid structure [6-9]. This microstructured SERS substrate has a large surface area, therefore increases the number of effective hot spots, and further enhances the sensitivity of the SERS signal [10]. The microstructured silicon pyramid is particularly interesting one. Pyramid microstructure has been used in solar cells to improve the conversion efficiency of solar cells through its light trapping effect [11-13]. Due to its low process threshold and cost-effective performance, this is the most mature production process in the photovoltaic industry for decades. An anisotropic wet chemical etch is typically utilized to form a micro-sized pyramidal structure as a light-harvesting structure using different etch rates of silicon crystals at $<100>$ and $<111>$ surfaces [14]. It has been shown that a randomly sized pyramid array surface can have better light-harvesting efficiency and therefore a better absorption coefficient than planar and single sized pyramid array surfaces [15]. In some SERS studies [7-9], It has been proven that the Si pyramids can increase the amount of the hot spots by providing large surface area and create strong local electric field [9]. However, substrates with only 3D microstructures are not sufficient to generate strong SERS signals, and studies have shown that strong local surface plasmons (SERS hot spots) occur at the nano-gaps between noble metal nanostructures $[9,10]$. Therefore, it is preferred that the SERS substrate can have both micron and nanostructures, which will greatly increase the SERS signal strength.

In this study, we provide a facile method to produce SERS active substrates using simple steps and inexpensive costs. We use wet etching to create a pyramid-shaped large structure on a silicon wafer and then use the electrochemical process to create gold nanostructures on the pyramid surface. In previous studies, electrochemical surface treatment was mostly used to roughening the surface of bulk gold or bulk silver materials [16,17]. In this study, we applied the electrochemical oxidation and reduction cycles to the gold thin film on the silicon micro-pyramid array, which leads to atomic restructuring of the gold thin film surface. We studied the surface metal dissolution/redeposition effect of the electrochemical treatment to the gold thin film on the micro-pyramid array silicon substrate, and find a method to optimize the electrochemical treatment and maximized surface area of the gold thin film on the micro-pyramid array silicon substrate. We observed the electrochemical redox cycle curves and SERS signal of substrates, and found the relationship between electrochemical oxidation peaks and SERS signal strength. From the relationship between electrochemical oxidation peaks and SERS signal intensity, we obtained the best electrochemical treatment cycles for SERS substrate preparation. In addition, by combining the micro-pyramid array and gold nanostructure on the pyramid surface, this micro/nano hierarchical structured SERS substrate can greatly enhance the Raman signal. The SERS enhancement effect is tested with a low concentration of Rhodamine 6G (R6G). Finally, we applied SERS substrates prepared by optimized electrochemical parameters to the detection of R6G and malachite green solutions. The results show that the substrate has high sensitivity and can measure very low concentrations of R6G and malachite green.

\section{Methodology and Experiments}

\subsection{Fabrication of the Silicon Micro-Pyramid Structures}

To fabricate silicon micro-pyramid structures, we first cleaned the silicon wafer with ethanol and acetone. The silicon wafer was then etched at $85^{\circ} \mathrm{C}$ for $40 \mathrm{~min}$ in a mixed solution of $\mathrm{NaOH}(1.8 \mathrm{wt} \%)$, water and isopropyl alcohol (IPA, $5 \mathrm{vol} \%$ ). During the $\mathrm{NaOH}$ etching, the etching solution was stirred using a magnetic stirrer, and the rotational speed of the magnetic stirring was set to $200 \mathrm{rpm}$. After etching, the wafer was rinsed with deionized water and dried with nitrogen. 


\subsection{Fabrication of the Hierarchical Micro/Nanostructure}

To create a hierarchical micro/nanostructure, a gold thin film with a thickness of $100 \mathrm{~nm}$ was first deposited on micro-pyramids using a dc magnetron sputtering system (Cressington 108 Sputter Coater). The wafer with the gold film deposited was diced into $15 \times 15 \mathrm{~mm}$ chips before the electrochemical treatment. The chip was then attached to a glass slide and wire connected as shown in Figure 1c. The conductive wire was covered with silicone to prevent reaction with electrolyte. The chip (working electrode) was then immersed in the KCL solution along with a counter electrode (platinum) and a reference electrode $(\mathrm{Ag} / \mathrm{AgCl})$. The sample was then electrochemically treated by applied oxidation and reduction cycles to form the nanostructure on the surface of the gold thin film. The electrochemical treatment was performed by using a VidaBio VS1 electrochemical potentiostat with a voltage sweep range of $-800 \mathrm{mV}$ to $800 \mathrm{mV}$, voltage sweep rate: $50 \mathrm{mV} / \mathrm{s}$, oxidation and Reduction cycles from 1 to 15 cycles in a KCL solution with a concentration of $0.2 \mathrm{~mol} / \mathrm{L}$. The micro-pyramids combined with the nanostructured gold thin film formed the hierarchical micro/nanostructure. The fabrication process of the described micro/nano hierarchical structure is illustrated in Figure 1.

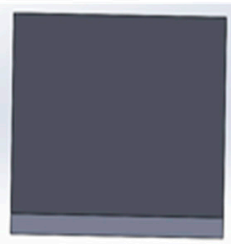

Silicon wafer

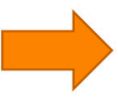

(a). Wet Etch $(\mathrm{NaOH})$

\section{Silicon wafer with} micro-pyramid

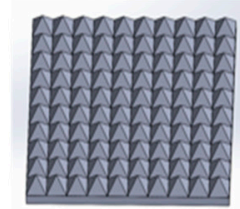

(b). Gold thin film deposition

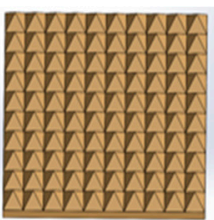

Gold thin film on top of silicon micro-pyramid

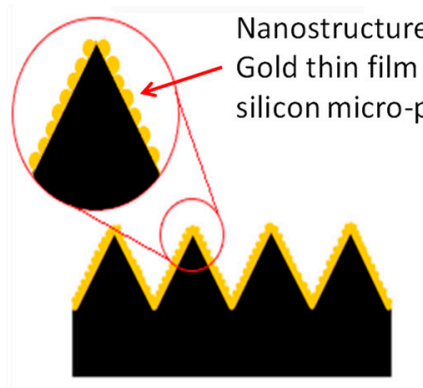

Nanostructured Gold thin film on silicon micro-pyramid

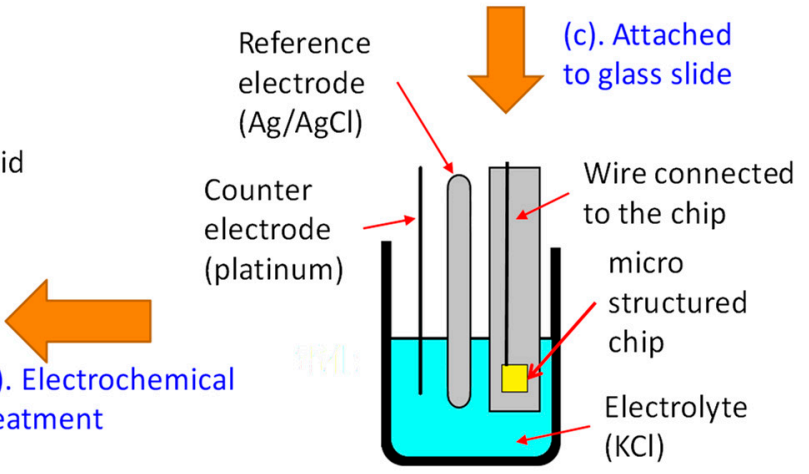

Figure 1. The fabrication process of the described micro/nano hierarchical structure. (a) The silicon wafer was wet etched in a mixed solution of $\mathrm{NaOH}$, water, and isopropyl alcohol. (b) Depositing a gold thin film on the micro-pyramid array using a sputtering system. (c) Attach the chip to a slide before electrochemical treatment. (d) The sample was treated with electrochemical oxidation and reduction cycles to create nanostructures on the surface of the gold thin film.

\subsection{Experiments}

The surface morphology of the fabricated SERS substrate was investigated by using a scanning electron microscope (SEM, JSM-7800F, JEOL Ltd., Tokyo, Japan) and an atomic force microscope (AFM, Bruker Dimension Icon, Bruker Corporation, Billerica, USA). The SERS performances of fabricated substrates were tested with R6G and malachite green solutions. Five microliters of different concentrations of R6G aqueous solution and malachite green solution were separately dropped onto the prepared SERS substrates and allowed to dry at room temperature for Raman spectroscopy measurements. SERS spectra were measured by a microscope Raman spectrometer (Nanofinder 30, Tokyo Instruments, Tokyo, Japan) under excitation of a $632.8 \mathrm{~nm}$ HeNe laser, wherein the effective power of the laser source was set to $0.1 \mathrm{~mW}$. The diameter of the focused laser spot on the surface of 
the sample was approximately $2 \mu \mathrm{m}$, and the integration time per measurement was $10 \mathrm{~s}$. The SERS substrate performance was then characterized by calculating the SERS enhancement factor.

\section{Results and Discussion}

\subsection{Characterization of the Developed Substrates}

The SEM image showed in Figure 2a is the anisotropic wet etched silicon micro-pyramid array structure. From the image, one can also see that the produced micro-pyramid array is a randomly sized pyramid array surface. This feature may be an advantage, as mentioned in [15], a random-sized pyramid array surface can have better light collection efficiency than a planar and single-sized pyramid array surface over a wide wavelength range. The average spacing between the micro-pyramids is about $2.8 \mu \mathrm{m}$, and the average width of the micro-pyramids at the base is about $1.4 \mu \mathrm{m}$. The AFM image in Figure $2 b$ indicates that the average height of these micro-pyramids is about $2 \mu \mathrm{m}$.

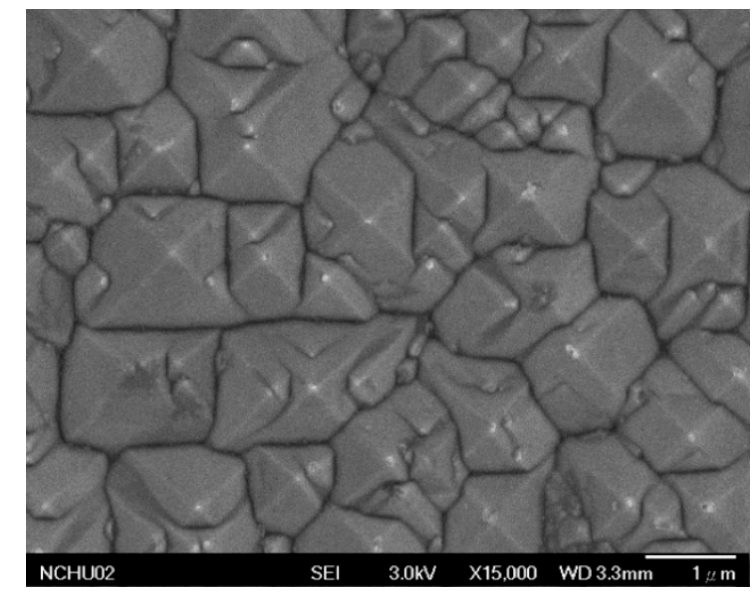

(a)

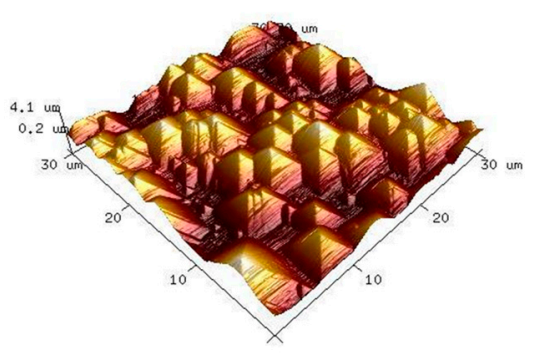

(b)

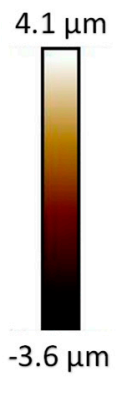

Figure 2. (a) The SEM image of pyramid microstructure formed by anisotropic wet etching. (b) The pyramid size measured by an atomic force microscope (AFM), the overall pyramid average height is 2 $\mu \mathrm{m}$, and the average spacing is $2.8 \mu \mathrm{m}$.

A gold film with a thickness of $100 \mu \mathrm{m}$ was then deposited on the micro-pyramid array by sputtering and then electrochemical treated to form nanoparticles on the gold film surface. The size of these nanoparticles was controlled by electrochemical redox cycles. Figure 3a-d show SEM images of these gold nanoparticles with different electrochemical redox cycles of $0,5,10$, and 15 , respectively.

As can be seen from these figures, the morphology of the gold film on the surface of the micro-pyramid structure after electrochemical treatment clearly shows the formation of many tiny nano-grain structures. It can be seen that the longer the duration, the larger the diameter of these nanoparticles. In the first 10 redox cycles, the diameter of these nanoparticles has relatively faster increasement, and the nanoparticles become larger and larger. After 10 redox cycles, the nanoparticles' changes are not obvious. However, if too many redox cycles are performed, the gold film will become too thin and expose the silicon pyramid (as shown in Figure 4). 


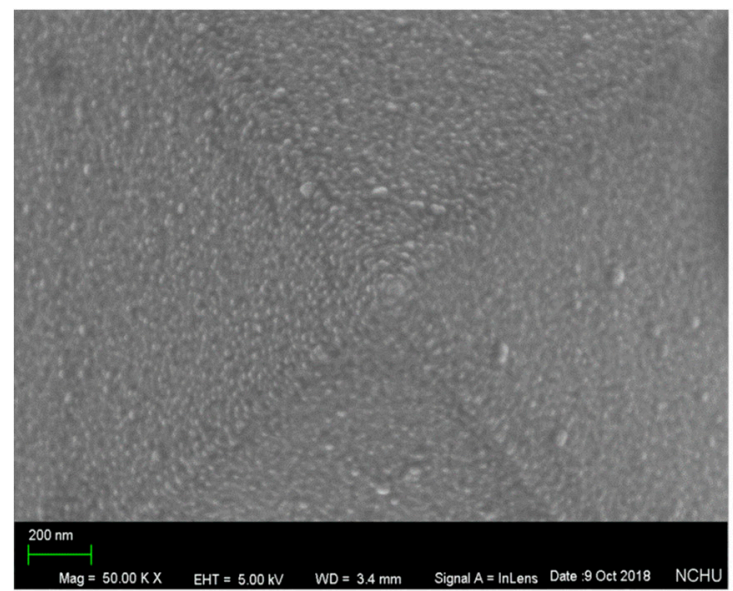

(a)

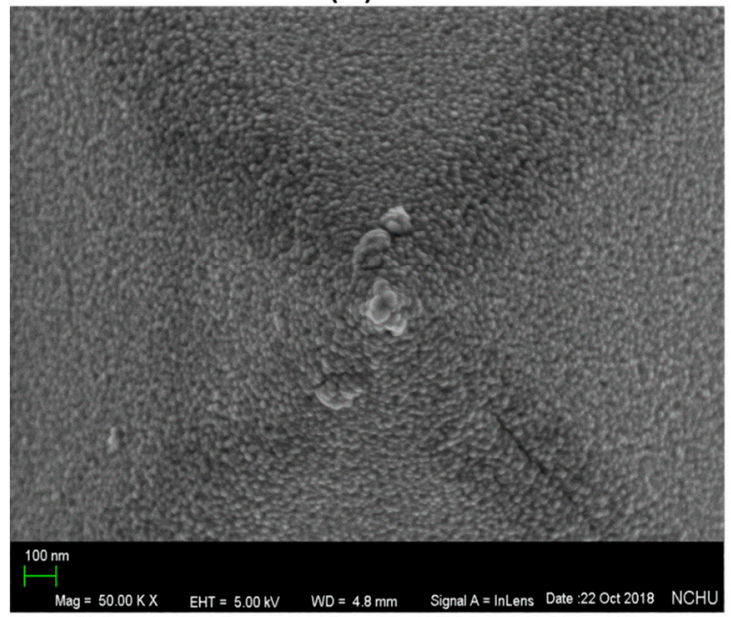

(b)

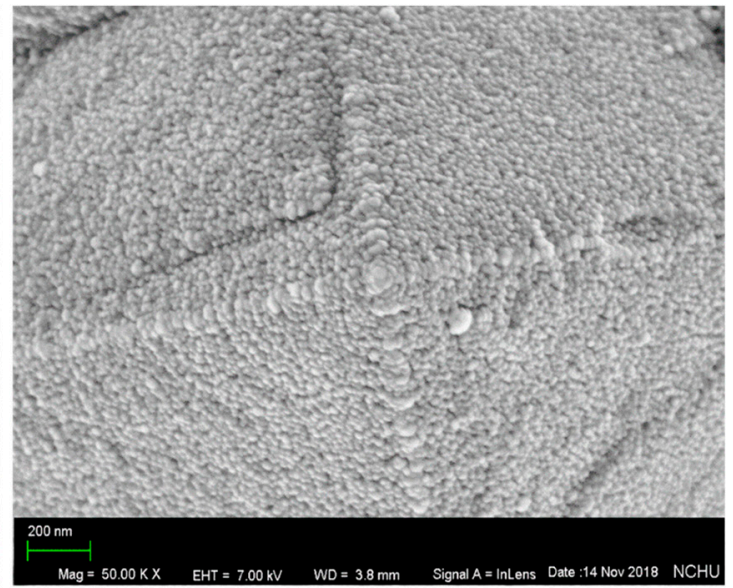

(c)

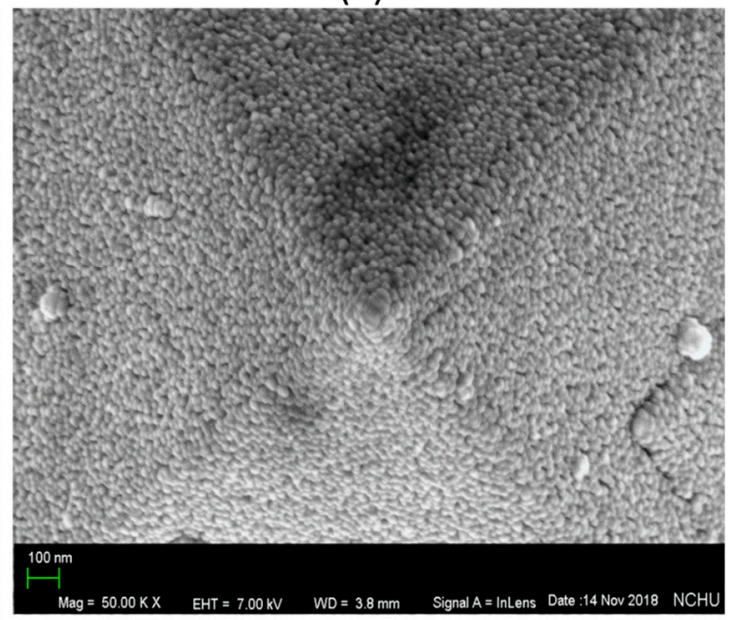

(d)

Figure 3. (a) The gold film morphology on the surface of the micro-pyramid structure before electrochemical treatment, the surface is relatively smooth (compared with the electrochemically treated surface). (b) The gold film morphology after 5 redox cycles. (c) The gold film morphology after 10 redox cycles. (d) The gold film morphology after 15 redox cycles.

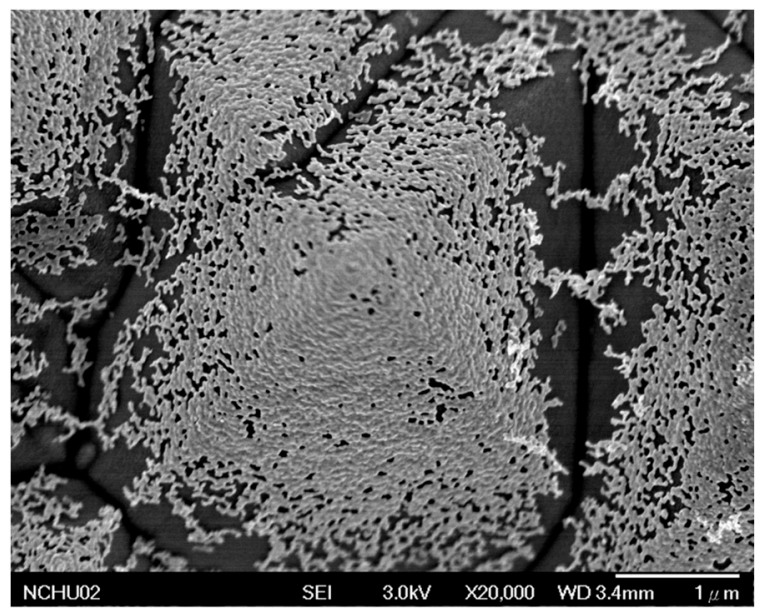

Figure 4. The gold film on the surface of the pyramid is oxidized and corroded. After too many oxidization-reduction cycles, the gold film became too thin and exposed the pyramid. 
Figure $3 c$,d shows that the diameter of the nanoparticles is about $20 \mathrm{~nm}$ when the redox cycle is 10-15 cycles. The gap between the nanoparticles is in the range of a few nanometers and is suitable for producing many intense hot spots. Therefore, it is very helpful to enhance the SERS effect. Figure 4 shows that when the redox cycle was set to 30 cycles, the gold film became too thin and exposed the underlying pyramid.

When the gold film on the surface of the pyramid is subjected to cyclic voltammetry redox scanning, the gold on the surface is first oxidized into the solution and then reduced to the surface of the gold film. During this process, the gold surface is granulated to form many gold nano-gaps. In the experiment, we repeated the cyclic voltammetry redox scanning cycle multiple times. Figure 5 shows the cyclic voltammetry curves from the 1st scan to the 15 th scan cycle in a $0.2 \mathrm{M} \mathrm{KCl}$ solution at a scan rate of $50 \mathrm{mV} \mathrm{s}^{-1}$. The position of the blue arrow in the figure shows the position where the oxidation peak occurs, which is about $530 \mathrm{mV}$. The oxidation peak currents were further plotted against the scanning cycles in Figure 6. As can be seen from Figures 5 and 6, when the number of scanning cycles increased, the peak current of the oxidation also increased. When it reaches a certain amount of cycles (in this case around 10 cycles), the oxidation peaks reach a plateau state. The change in the microscopic surface area of the electrode produced by the oxidation-reduction cycles is proportional to the oxidation peak, as Daubinger and Netzahualcóyotl mentioned in [18,19]. Therefore, a higher oxidation peak indicates that more nanostructures were produced on the electrode surface during the oxidation-reduction scanning process.

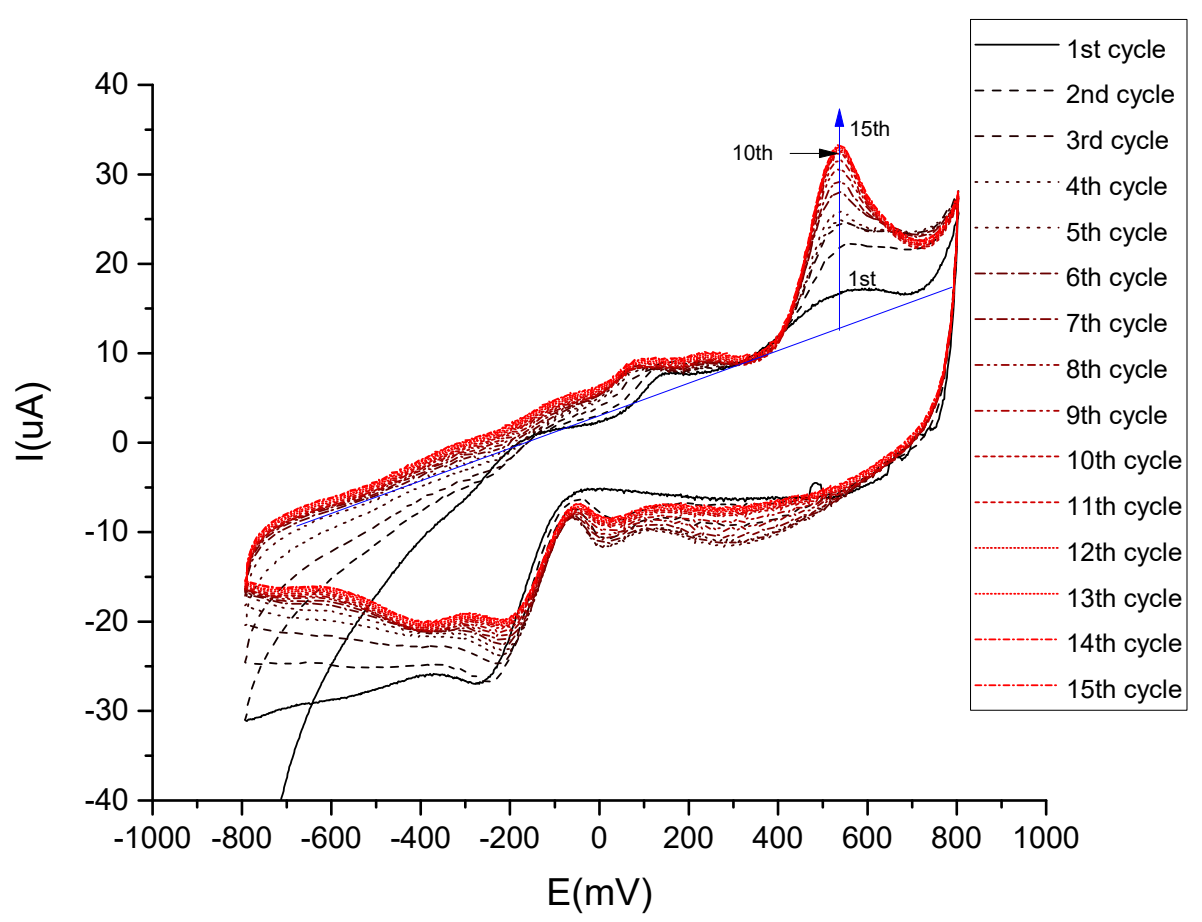

Figure 5. The cyclic voltammetry curves from the 1st scan to the 15th scan cycle at a sweeping rate of $50 \mathrm{mV} \mathrm{s}^{-1}$ in the $0.2 \mathrm{M} \mathrm{KCl}$ solution. Each cycle curve was plotted in a different line style for clarity. The position of the blue arrow in the figure shows the position where the oxidation peak occurs, which is about $530 \mathrm{mV}$. We can see from the figure that the oxidation peak current increases with the number of scanning cycles. However, the increment gradually reduces, and its increment was quite limited after the number of scanning cycles exceeded 10. 


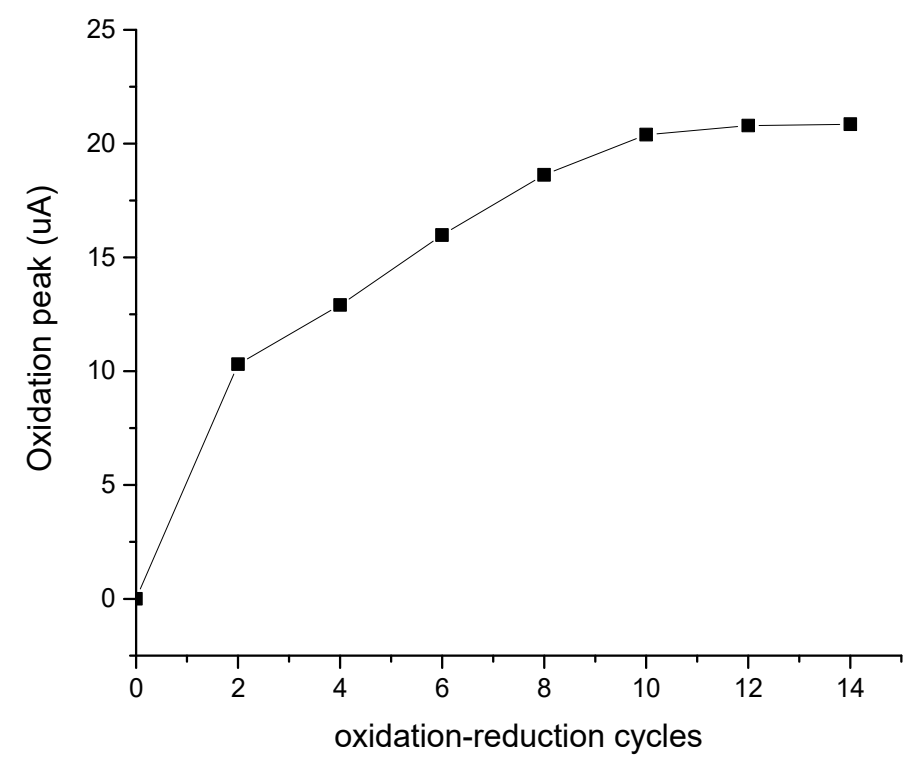

Figure 6. The oxidation peak currents at $530 \mathrm{mV}$ were plotted against the scanning cycles. It can be seen that the oxidation peak current increases with the number of scanning cycles. The increment gradually reduces, and when the voltammetry redox scan reaches a certain amount of cycles (in this case around 10 cycles), the oxidation peaks reach a plateau state.

SERS measurements of substrates electrochemically treated with different oxidation-reduction cycles were conducted for comparison. From the SERS spectra of $1 \mu \mathrm{M}$ R6G solution measured by substrates with different oxidation-reduction cycle times $(0,2,4,6,8,10,12$, and 14, respectively), the SERS characteristic peak intensity of R6G at $1510 \mathrm{~cm}^{-1}$ increase rapidly in the first few cycles and reach a plateau after 10 cycles (as shown in Figure 7). It has the same tendency as the oxidation peak of the redox curve shown in Figure 6. This is because higher oxidation peaks indicate more reaction surface area, which means more nanostructures were created. More nanostructures not only have a larger reaction area but also produce higher density SERS hot spots and lead to higher SERS intensities. Therefore, the optimal number of oxidation-reduction cycles can be determined by monitoring the oxidation-reduction plots. Once it reaches a plateau state, it means the optimal oxidation-reduction cycles for SERS enhancement has been reached.

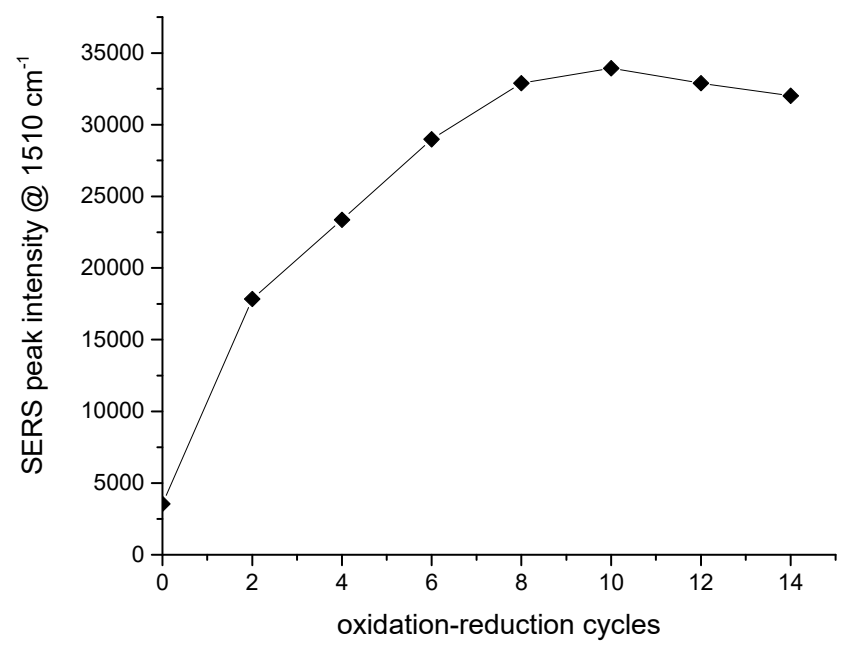

Figure 7. It shows the surface-enhanced Raman scattering (SERS) experiments results of $1 \mu \mathrm{M}$ Rhodamine 6 (R6G) solution measured by SERS chips with different oxidation-reduction cycles $(0,2,4$, $6,8,10,12$, and 14 , respectively). It can be seen that in the first few cycles, the intensity of the SERS characteristic peak of $\mathrm{R} 6 \mathrm{G}$ at $1510 \mathrm{~cm}^{-1}$ increased rapidly and reached a plateau after 10 cycles. 
Another note worth mentioning is that the peak current of the reduction is less than the peak oxidation current, which means that the redox process is irreversible. Therefore, the gold film on the surface of the pyramid is gradually oxidized and dissolved. This will cause the gold film on the surface of the pyramid to gradually decrease. This is why too many redox scan cycles can cause the gold film to become too thin and expose the underlying pyramid (as shown in Figure 4). Therefore, we need to carefully monitor the oxidation-reduction curves and stop the oxidation-reduction process once the oxidation peak reaches a plateau.

\subsection{Simulations}

Simulations were performed to further understand the effects of gold nanoparticles decorated micro-pyramid array substrate. We calculated the local electric field of the gold nanoparticles decorated micro-pyramid structure using a commercial the finite element method (FEM) software (COMSOL Multiphysics). We set the diameter of gold nanoparticles to $20 \mathrm{~nm}$ and pitch between nanoparticles to 22 $\mathrm{nm}$. Figure 8 shows a cross-sectional view of the electric field distribution between two micro-pyramids with an incident light wavelength of $633 \mathrm{~nm}$. In this figure, the normalized electric field represents the ratio between the electric field excited by the local surface plasmon resonance and the background electric field. We can clearly see that a strong electric field is formed around gold nanoparticles, especially between nanoparticles. V-shaped caves formed between the pyramids can also help to enhance the electric field by concentrating the input of light energy. This micro/nano hierarchical structured substrate helps to form many strong hot spots. Since the SERS enhancement effect is proportional to the fourth power of the local electric field intensity [20], these hot spots have a very good enhancement effect on the SERS signal.

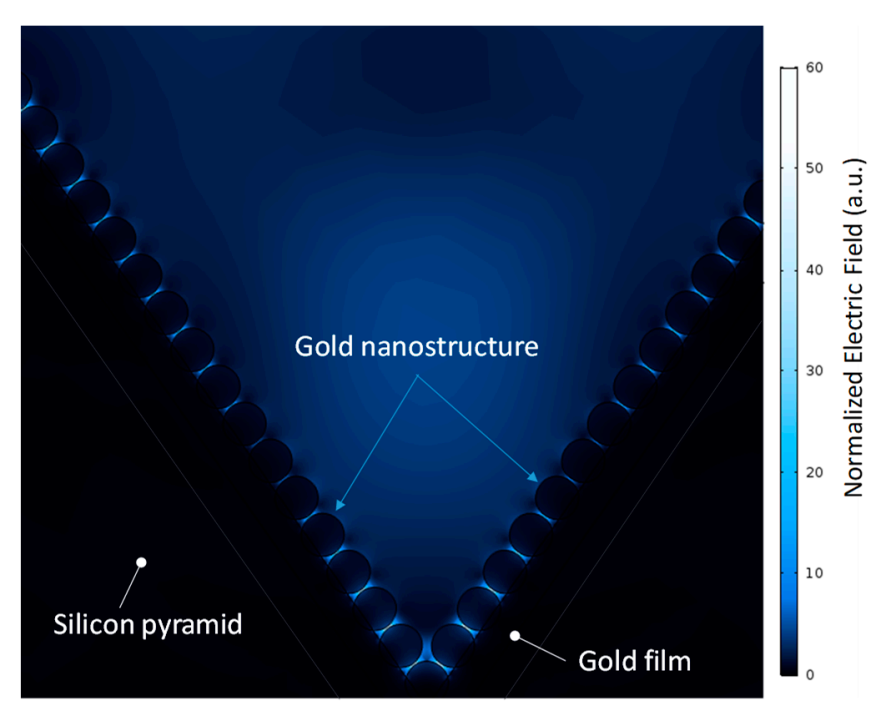

Figure 8. A cross-sectional view of the electric field distribution between two micro-pyramids with an incident light wavelength of $633 \mathrm{~nm}$. In this figure, the normalized electric field represents the ratio between the electric Field excited by the local surface plasmon resonance and the background electric field.

\subsection{SERS Measurements}

Figure 9 shows the SERS spectra of R6G solutions with concentrations of $1 \times 10^{-6} \mathrm{M}, 1 \times 10^{-7}$ $\mathrm{M}, 1 \times 10^{-8} \mathrm{M}$, and $1 \times 10^{-9} \mathrm{M}$, respectively, using our SERS substrate with 10 oxidation-reduction cycles. The Raman band assignments of the main peaks for R6G were given in Figure 9. Even at a low concentration of $1 \mathrm{nM}$, characteristic peaks of R6G at 607, 765, 1176, 1304, 1355, 1503, and $1640 \mathrm{~cm}^{-1}$ were clearly observed from these spectra. These spectra indicate that our SERS substrates have a good enhancement factor. 


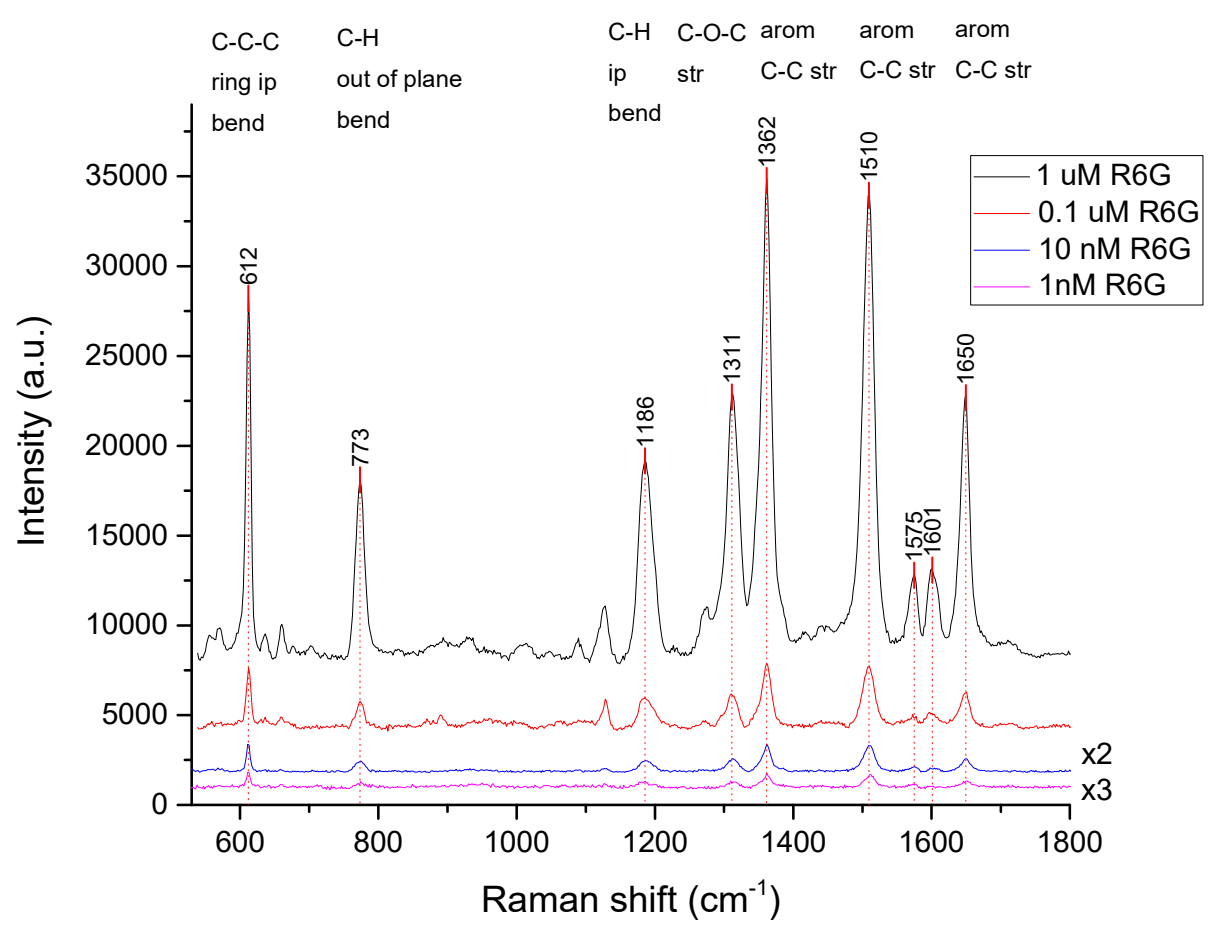

Figure 9. Shows the SERS spectra of R6G solutions with concentrations of $1 \times 10^{-6} \mathrm{M}, 1 \times 10^{-7} \mathrm{M}, 1 \times$ $10^{-8} \mathrm{M}$, and $1 \times 10^{-9} \mathrm{M}$, respectively, using our SERS substrate with 10 oxidation-reduction cycles.

We used the following well-established formula [21] to estimate the enhancement factor (EF) value of R6G:

$$
E F=\left(I_{S E R S} / I_{b u l k}\right) /\left(N_{b u l k} / N_{S E R S}\right)
$$

where $I_{S E R S}$ is the vibrational intensity of the SERS of the R6G molecule at $1510 \mathrm{~cm}^{-1}$, and $I_{b u l k}$ is the vibrational intensity of the normal Raman spectrum of the R6G molecule at $1510 \mathrm{~cm}^{-1}$. $N_{S E R S}$ is the number of molecules irradiated by laser spots under SERS, and $N_{b u l k}$ is the number of molecules of the bulk sample irradiated by laser spots under normal Raman condition. The EF was calculated to be about $1 \times 10^{7}$, which is quite good for a gold-based SERS substrate [21,22]. This result is assuming the SERS substrate is densely packed with R6G molecules after immersed in $10^{-6} \mathrm{M}$ R6G solution for $12 \mathrm{~h}$. However, the R6G is likely to cover the substrate at a lower density. Therefore, the SERS enhancement factor calculated here is the lower limits.

The developed SERS substrates were also tested with low concentration malachite green solution. Figure 10 shows measured SERS spectra for malachite green concentrations ranging from $0.01 \mathrm{nM}$ to $10 \mathrm{nM}$ on SERS substrates. The Raman characteristic peaks of malachite green at $1620 \mathrm{~cm}^{-1}, 1370 \mathrm{~cm}^{-1}$, $1175 \mathrm{~cm}^{-1}, 916 \mathrm{~cm}^{-1}$, and $802 \mathrm{~cm}^{-1}$ are in-plane $C-C$ stretching vibration of the ring, overlapping effect of in-plane $\mathrm{N}$-phenyl stretching vibration and in-plane $\mathrm{C}-\mathrm{C}$ stretching vibration, in-plane aromatic $\mathrm{C}-\mathrm{H}$ vibration, ring-breathing, and out-of-plane phenyl-H bending, respectively. These characteristic peaks of malachite green are clearly observed from the spectra. According to the result, the SERS substrates were found to be able to detect a low concentration of $0.01 \mathrm{nM}$ malachite green solution, which is very close to the finding in previous studies $[23,24]$. The enhancement factors for different concentrations of malachite green were also calculated. The Raman characteristic peak values of malachite green at $1370 \mathrm{~cm}^{-1}$ acquired from Figure 10 were used to calculate the enhancement factors for different concentrations of malachite green. The resulting enhancement factors for concentrations of $10 \mathrm{nM}, 1 \mathrm{nM}, 0.1 \mathrm{nM}$, and $0.01 \mathrm{nM}$ malachite green are $1.21 \times 10^{7}, 1.49 \times 10^{7}, 7.81 \times 10^{7}$, and $4.05 \times$ $10^{8}$, respectively. It can be seen that when the concentration of the MG solution to be measured is lower than $1 \mathrm{nM}$, as the concentration decreases, the enhancement factor (or sensitivity) of the developed chip becomes larger, rather than a constant value. 


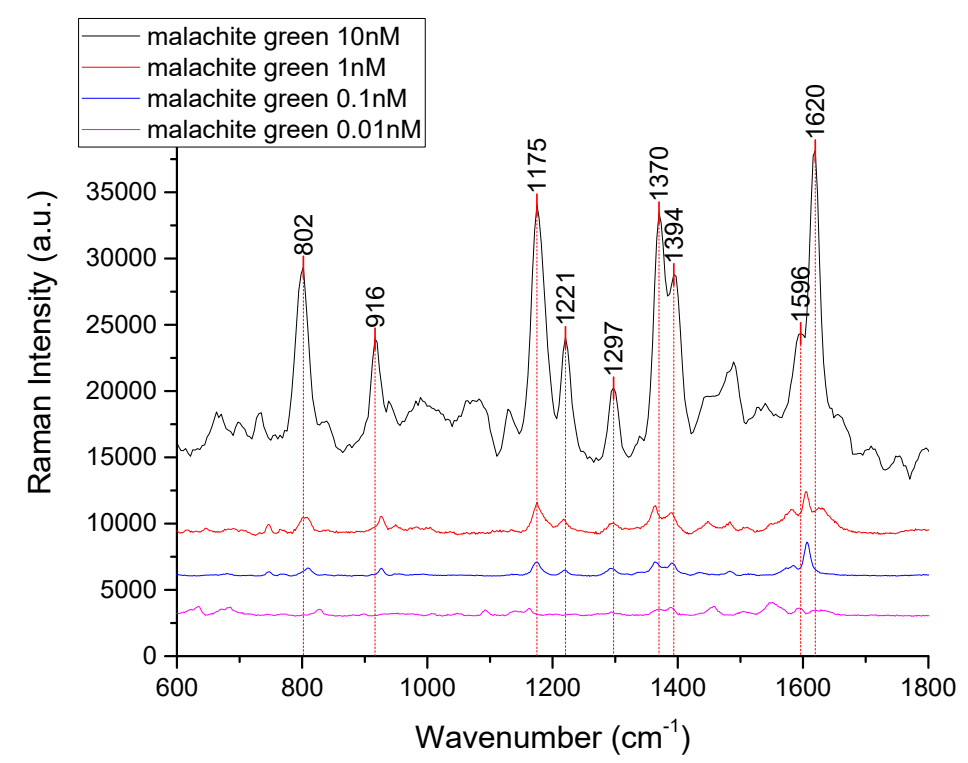

Figure 10. The figure shows measured SERS spectra for malachite green concentrations ranging from $0.01 \mathrm{nM}$ to $10 \mathrm{nM}$ on SERS substrates.

\section{Conclusions}

In conclusion, we have presented a facile method for synthesizing hierarchical micro/nanostructured SERS active substrates using simple steps and inexpensive costs. The SERS active substrates were fabricated through wet etching to create a pyramid-shaped large structure on a silicon wafer and then used electrochemical treatments to create gold nanostructure on the pyramid surface. From the relationship between electrochemical oxidation peaks and SERS signal intensity, we optimized the electrochemical treatment for SERS substrate preparation. Simulation has shown that many strong hot spots can be formed between the nano-gap of gold nanostructures. According to the SERS experiments, the micro/nano hierarchical structured SERS substrate showed a strong SERS enhancement factor up to $1 \times 10^{7}$. The developed SERS substrates were further applied to the detection of malachite green solutions. The results showed that the substrate has high sensitivity and can measure very low concentrations of malachite green $(0.01 \mathrm{nM})$, indicating its practical application potential in food contamination detection. Therefore, the developed SERS active substrate is highly promising for use in rapid chemical and biomolecular detection applications, including medicine, food safety, and biotechnology.

Author Contributions: Conceptualization, C.-Y.H.; methodology, C.-Y.H.; investigation, C.-H.C.; data curation, C.-H.C.; writing — original draft preparation, C.-Y.H.; writing—review and editing, C.-Y.H.; supervision, C.-Y.H.; project administration, C.-Y.H.; funding acquisition, C.-Y.H.

Funding: This research was funded by Ministry of Science and Technology, Taiwan, grant number MOST 107-2221-E-005-045.

Acknowledgments: The authors would like to thank the Ministry of Science and Technology of the Republic of China, Taiwan, for financially supporting this research under Contract No. Grant MOST 107-2221-E-005-045.

Conflicts of Interest: The authors declare no conflict of interest.

\section{References}

1. Andersen, W.C.; Turnipseed, S.B.; Roybal, J.E. Quantitative and confirmatory analyses of malachite green and leucomalachite green residues in fish and shrimp. J. Agric. Food Chem. 2006, 54, 4517-4523. [CrossRef] [PubMed]

2. Deng, Y.; Chu, D. Coherence properties of different light sources and their effect on the image sharpness and speckle of holographic displays. Sci. Rep. 2017, 7, 1-12. [CrossRef] [PubMed] 
3. Chemicals in Food: Safety Controls-GOV.UK. Available online: https://www.gov.uk/guidance/chemicalsin-food-safety-controls (accessed on 1 November 2019).

4. Laboratory Information Bulletin (LIB) 4363: Malachite Green and Leucomalachite Green in Fish and Shrimp|FDA. Available online: https://www.fda.gov/food/laboratory-methods-food/laboratoryinformation-bulletin-lib-4363-malachite-green-and-leucomalachite-green-fish-and-shrimp (accessed on 26 September 2019).

5. Akin, M.S.; Yilmaz, M.; Babur, E.; Ozdemir, B.; Erdogan, H.; Tamer, U.; Demirel, G. Large area uniform deposition of silver nanoparticles through bio-inspired polydopamine coating on silicon nanowire arrays for practical SERS applications. J. Mater. Chem. B 2014, 2, 4894-4900. [CrossRef]

6. Wang, Y.; Lu, N.; Wang, W.; Liu, L.; Feng, L.; Zeng, Z.; Li, H.; Xu, W.; Wu, Z.; Hu, W.; et al. Highly effective and reproducible surface-enhanced Raman scattering substrates based on Ag pyramidal arrays. Nano Res. 2013, 6, 159-166. [CrossRef]

7. Xiu, X.; Guo, Y.; Li, C.; Li, Z.; Li, D.; Zang, C.; Jiang, S.; Liu, A.; Man, B.; Zhang, C. High-performance 3D flexible SERS substrate based on graphene oxide/silver nanoparticles/pyramid PMMA. Opt. Mater. Express 2018, 8, 844. [CrossRef]

8. Chen, S.; Liu, B.; Zhang, X.; Mo, Y.; Chen, F.; Shi, H.; Zhang, W.; Hu, C.; Chen, J. Electrochemical fabrication of pyramid-shape silver microstructure as effective and reusable SERS substrate. Electrochim. Acta 2018, 274, 242-249. [CrossRef]

9. Li, Z.; Xu, S.C.; Zhang, C.; Liu, X.Y.; Gao, S.S.; Hu, L.T.; Guo, J.; Ma, Y.; Jiang, S.Z.; Si, H.P. High-performance SERS substrate based on hybrid structure of graphene oxide/AgNPs/Cu film@pyramid Si. Sci. Rep. 2016, 6, 38539. [CrossRef]

10. Xu, J.; Li, C.; Si, H.; Zhao, X.; Wang, L.; Jiang, S.; Wei, D.; Yu, J.; Xiu, X.; Zhang, C. 3D SERS substrate based on Au-Ag bi-metal nanoparticles/MoS 2 hybrid with pyramid structure. Opt. Express 2018, 26, 21546. [CrossRef]

11. Campbell, P.; Green, M.A. Light trapping properties of pyramidally textured surfaces. J. Appl. Phys. 1987, 62, 243-249. [CrossRef]

12. Mahmoud Al, A.; Lahlouh, B. Silicon Pyramid Structure as a Reflectivity Reduction Mechanism. J. Appl. Sci. 2017, 17, 374-383. [CrossRef]

13. Zhang, C.; Chen, L.; Zhu, Y.; Guan, Z. Fabrication of 20.19\% Efficient Single-Crystalline Silicon Solar Cell with Inverted Pyramid Microstructure. Nanoscale Res. Lett. 2018, 13, 91. [CrossRef] [PubMed]

14. Wang, X.; Yang, Z.; Gao, P.; Yang, X.; Zhou, S.; Wang, D.; Liao, M.; Liu, P.; Liu, Z.; Wu, S.; et al. Improved optical absorption in visible wavelength range for silicon solar cells via texturing with nanopyramid arrays. Opt. Express 2017, 25, 10464. [CrossRef] [PubMed]

15. Zhong, S.; Wang, W.; Zhuang, Y.; Huang, Z.; Shen, W. All-Solution-Processed Random Si Nanopyramids for Excellent Light Trapping in Ultrathin Solar Cells. Adv. Funct. Mater. 2016, 26, 4768-4777. [CrossRef]

16. Wain, A.J.; O'connell, M.A. Advances in surface-enhanced vibrational spectroscopy at electrochemical interfaces. Adv. Phys. X 2017, 2, 188-209. [CrossRef]

17. Wang, W.; Huang, Y.F.; Liu, D.Y.; Wang, F.F.; Tian, Z.Q.; Zhan, D. Electrochemically roughened gold microelectrode for surface-enhanced Raman spectroscopy. J. Electroanal. Chem. 2016, 779, 126-130. [CrossRef]

18. Arroyo-Currás, N.; Scida, K.; Ploense, K.L.; Kippin, T.E.; Plaxco, K.W. High Surface Area Electrodes Generated via Electrochemical Roughening Improve the Signaling of Electrochemical Aptamer-Based Biosensors. Anal. Chem. 2017, 89, 12185-12191. [CrossRef] [PubMed]

19. Daubinger, P.; Kieninger, J.; Unmüssig, T.; Urban, G.A. Electrochemical characteristics of nanostructured platinum electrodes-A cyclic voltammetry study. Phys. Chem. Chem. Phys. 2014, 16, 8392-8399. [CrossRef]

20. Moskovits, M. Surface-enhanced Raman spectroscopy: A brief retrospective. J. Raman Spectrosc. 2005, 36, 485-496. [CrossRef]

21. Litti, L.; Meneghetti, M. Predictions on the SERS enhancement factor of gold nanosphere aggregate samples. Phys. Chem. Chem. Phys. 2019, 21, 15515-15522. [CrossRef]

22. He, Y.; Song, C.; Que, L. Nanoforest-based SERS sensor fabricated using a maskless process for detecting chemical and pathogen. Microsyst. Technol. 2019, 25, 4349-4356. [CrossRef] 
23. Song, J.; Huang, Y.; Fan, Y.; Zhao, Z.; Yu, W.; Rasco, B.; Lai, K. Detection of Prohibited Fish Drugs Using Silver Nanowires as Substrate for Surface-Enhanced Raman Scattering. Nanomaterials 2016, 6, 175. [CrossRef] [PubMed]

24. Polavarapu, L.; Porta, A.L.; Novikov, S.M.; Coronado-Puchau, M.; Liz-Marzán, L.M. Pen-on-Paper Approach toward the Design of Universal Surface Enhanced Raman Scattering Substrates. Small 2014, 10, 3065-3071. [CrossRef] [PubMed]

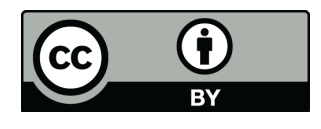

(C) 2019 by the authors. Licensee MDPI, Basel, Switzerland. This article is an open access article distributed under the terms and conditions of the Creative Commons Attribution (CC BY) license (http://creativecommons.org/licenses/by/4.0/). 